\title{
Validación de la Environmental Reward Observation Scale (EROS) en población colombiana
}

\section{Validation of the Environmental Reward Observation Scale (EROS) in Colombian population}

Recepción: 31 Agosto 2015 | Aprobación: 01 Agosto 2016

\author{
Mario Andrés Valderrama-Díaz** \\ Fundación Universitaria Konrad Lorenz, Colombia \\ Javier Mauricio Bianchi-Salguero*** \\ Fundación Universitaria Konrad Lorenz, Colombia \\ Javier Andrés Villalba-Garzón**** \\ Fundación Universitaria Konrad Lorenz, Colombia
}

*Teléfono: 3014498039. Correos electrónicos: marioandresvalderramad@mail.com; marioa.valderramad@konradlorenz.edu.co

**Teléfono: 3008664930. Correos electrónicos: biancosal@gmail.com; javierm.bianchis@konradlorenz.edu.co

***Teléfono: 3134841290. Correos electrónicos: javierandresvillalba@gmail.com/ javiera.villalbag@konradlorenz.edu.co

\section{RESUMEN}

Se estimaron las propiedades psicométricas de la Environmental Reward Observation Scale (EROS) en población colombiana, con dos muestras: población comunitaria y clínica. Para la primera, se evaluaron 507 personas en 22 departamentos del país, con edades entre 18 y 72 años, $60.5 \%$ mujeres y el restante hombres; la segunda muestra estuvo compuesta por 68 personas, con edades comprendidas entre 18 y 72 años, atendidas en el momento de la evaluación por profesionales en salud mental. Se realizó la aplicación de los instrumentos (Enviromental Reward Observation Scale y Escala Autoaplicada de Depresión de Zung), a través de redes sociales y correos electrónicos, con el fin de tener una muestra más amplia y una mayor variabilidad de la misma. Se encontraron niveles apropiados de consistencia interna (alfa de 0.87) e importantes evidencias de validez de constructo (agrupación en un factor que explica el $46.82 \%$ de la varianza total) y discriminante $(r=-0.67)$.

Palabras clave

Environmental Reward Observation Scale, psicometría, evidencias de validez .

\section{ABSTRACT}

In the present study the psychometric properties of the Environmental Reward Observation Scale (EROS) in Colombian population were estimated with two different samples: population and clinic. For the first sample 517 people were evaluated in 22 departments of the country aged 18 to $72,60.5 \%$ are women and the remaining men, the second sample was composed of 68 people aged between 18 and 72 years who They are served at present by mental health professionals. Based on the above, the application of the instruments (Environmental Observation Reward Scale Y Auto Scale Applied Zung Depression) was made through social networks and emails in order to have a larger sample and greater variability the same in relation to the general population. Appropriate levels of internal consistency (alpha of 0.87) and significant evidence of construct validity (grouping by a factor that explains the $46.815 \%$ of the total variance) and discriminant $(r=-0.67)$.

Keywords

Environmental Reward Observation Scale, psychometrics, validity testing . 
Mario Andrés Valderrama-Díaz, Javier Mauricio Bianchi-Salguero, Javier Andrés Villalba-Garzón.

Para citar este artículo: Valderrama-Díaz, M. A., Bianchi-Salguero, J. M., \& Villalba- Garzón, A. A. (2016). Validación de la Environmental Reward Observation Scale (EROS) en población colombiana. Universitas Psychologica, 15 (4). http://dx.doi.org/10.11144/Javeriana.upsy15 -4 .vero

Existe un bajo número de desarrollos $\mathrm{y}$ validaciones de instrumentos que midan de manera eficiente los diferentes constructos en la psicología, este vacío es más evidente en lo que respecta a las terapias de tercera generación (Barraca-Mairal \& Pérez-Álvarez, 2010). Teniendo en cuenta esta falencia, es adecuado el desarrollo de estudios que estén relacionados con los procesos de adaptación de dichas escalas, en este punto radica el objetivo general de esta investigación, que consiste en evaluar las propiedades psicométricas de la Environmental Reward Observation Scale (EROS) en población colombiana.

Una de las primeras posturas conductualescontextuales para explicar los comportamientos depresivos fue la de Skinner (1953), quien menciona que los sentimientos como la depresión son subproductos respondientes, fruto de las interacciones conducta-ambiente. Otros modelos, asumen la presencia de conductas asociadas a la depresión con un carácter funcional. Estos modelos la consideran como una consecuencia de la falta de reforzamiento, ya sea en términos de una baja consistencia de reforzamiento positivo (Ferster, 1973) o de tasas bajas de reforzamiento positivo contingente a las respuestas (Lewinsohn, 1975). También se ha conceptualizado la depresión en términos del control aversivo o en términos de pérdida de contingencias debido al castigo (Seligman, 1975), además de mencionar la relación entre la erosión de los reforzadores y los déficits en habilidades con la presencia de esta conducta problema (Kanter, Mulick, Busch, Berlin, \& Martell, 2006).

Siguiendo la línea contextual, surge la Activación Conductual (AC) que explica la conducta depresiva en términos de un conjunto de comportamientos que se dan en función de un ambiente, cuyo efecto no depende de una entidad biológica o intrapsíquica, contemplando el contexto y la historia personal, como los elementos principales en su explicación (Peréz-Alvárez, 2007). La AC ha mostrado su efectividad como uno de los tratamientos más importantes para la depresión (Bianchi-Salguero \& Muñoz-Martínez, 2014), razón por la cual, el contar con estrategias de evaluación validadas, fortalece el marco empírico que apoya dicha intervención.

La AC centra su evaluación en las verbalizaciones del paciente sobre su comportamiento o registros de las actividades realizadas (Dimidjian, Martell, Addis, \& Herman-Dunn, 2008). Por medio de la implementación de las escalas, se puede aportar de manera significativa en la recolección de datos complementarios con el fin de establecer la validez y concordancia con los anteriores métodos de evaluación mencionados. Además, el uso de pruebas como el Behavioral Activation for Depression Scale ([BADS]; Kanter, Rusch, Busch, \& Sedivy, 2008) y la EROS permite estimar rápida, sencilla y fiablemente el nivel de activación y noción de reforzamiento ambiental a lo largo de todo el proceso terapéutico (BarracaMairal \& Pérez-Álvarez, 2010).

Como se hace evidente en investigaciones como la de Barraca-Mairal (2010) y HugoMendoza (2014), los estudios clínicos desde AC se encuentran ligados a las aplicaciones o medidas extraídas del Inventario de Depresion de Beck (BDI), situación que resulta contradictoria y poco objetiva, ya que la AC se centra en la interacción del sujeto con el medio, mientras que por su parte, el BDI evalúa dos dimensiones; una cognitivo-afectiva y otra somática (Beltrán, Freyre, \& Hernández-Guzmán, 2012) las cuales no se relacionan con los postulados de la AC. Por tal razón, el contar con una escala como la EROS, que mide la percepción o noción de refuerzo medioambiental, resulta más coherente con las estimaciones de la AC en la relación del organismo con el ambiente a través de relaciones funcionales tipo refuerzo, las cuales generarán un fortalecimiento de los procesos de intervención e investigación, manteniendo una coherencia 
epistemológica y teórica que apoye los diferentes procedimientos.

La Environmental Reward Observation Scale (EROS) es una escala que permite estimar la noción de refuerzo medioambiental. Este constructo se define como la capacidad que tiene el individuo para evaluar la disponibilidad y acceso a reforzadores en su contexto inmediato (Armento \& Hopko, 2007). En su versión original con población norteamericana, la escala presentó una alta consistencia interna (\# = 0.85), correlaciones ítem-test estadísticamente significativas $(\mathrm{p}<0.01$ ), rangos desde 0.55 hasta 0.8 , correlaciones moderadas con otras medidas de la depresión como el BDI = -0.69, el Cuestionario de Auto-Reporte de Síntomas Depresivos (CES-D) $=-0.65$, la Escala Autoaplicada para la Depresión de Zung (SDSZUNG) $=-0.54$ y el cuestionario "Qué tan deprimido está usted" $=-0.63$, sumado a la agrupación de todos los ítems en un factor, según el análisis factorial exploratorio. La adaptación española realizada por Barraca-Mairal y PérezÁlvarez (2010) presenta una alta consistencia interna $(\#=0.86)$ y se encontraron, como en el estudio original, correlaciones altas y significativas en la dirección esperada con medidas del BDI, Cuestionario de Aceptación y Acción (AAQ), Cuestionario de Pensamientos Automáticos (ATQ), Cuestionario de Ansiedad Estado-Rasgo (STAI) y el BADS, además de hallaron evidencias de validez divergente mediante la prueba t, la cual alcanzó un valor de -9.81 , con una alta significancia $(\mathrm{p}<0.001)$.

La EROS ha sido utilizada en investigaciones relacionadas con estados de ánimo bajos o diagnósticos de depresión (Barraca-Mairal, 2010; Carvalho et al., 2011; Gawrysiak, Nicholas, \& Hopko, 2009); además, de ser un instrumento necesario para medir noción de refuerzo medioambiental en investigaciones relacionadas con el consumo de tabaco y sustancias (Daughters, Magidson, Schuster, \& Safren, 2010; MacPherson et al., 2010), problemas de personalidad y trastornos esquizofrénicos (Daughters, Sargeant, Bornovalova, Gratz, \& LeJuez, 2008; Gamero, Vucinovich, \& Oñate, 2011), desarrollo de tareas en niños (Busch et al., 2009) y pacientes con problemas afectivos debido a enfermedades médicas como el cáncer (Carvalho \& Hopko, 2009; Carvalho et al., 2011; Ryba, LeJuez, \& Hopko, 2014).

El objetivo general de esta investigación fue estimar las propiedades psicométricas de la Environmental Reward Observation Scale (EROS) en población colombiana. Además, se plantearon como objetivos específicos los siguientes: identificar las evidencias de validez de contenido de la EROS en población colombiana, identificar las evidencias de validez de constructo de la EROS en población colombiana e identificar la consistencia interna de la prueba (confiablidad).

\section{Método}

\section{Tipo de estudio}

El estudio es de tipo instrumental-psicométrico. Según Montero y León (2007) los estudios instrumentales tienen como fin el desarrollo de instrumentos y pruebas, incluyendo tanto la adaptación o el diseño como el estudio de las propiedades psicométricas de los mismos. Este tipo de investigaciones buscan identificar las propiedades psicométricas, confiabilidad y validez de los instrumentos científicos de medida en ciencias sociales.

\section{Muestra poblacional}

La muestra estuvo conformada por 575 participantes seleccionados mediante un muestreo no probabilístico por conveniencia. Para la investigación, se incluyeron únicamente aquellas personas que participaron voluntariamente y diligenciaron las dos escalas utilizadas para el estudio. A diferencia de los usuales procesos de validación, se pretendió ampliar la población y no restringirse solamente a estudiantes universitarios, por tal razón, se tomaron datos de 22 departamentos del país. Del total de la muestra (575 participantes), el 
Mario Andrés Valderrama-Díaz, Javier Mauricio Bianchi-Salguero, Javier Andrés Villalba-Garzón.

$60.5 \%$ eran mujeres y el restante hombres; la edad promedio fue de 30.26 , oscilando entre 18 y 72 años y el $50 \%$ de los participantes tenía edades por encima de 28 años. Para el desarrollo del estudio, se contó con la participación de dos grupos denominados clínico y comunitario. Como criterios para la asignación al grupo clínico, se tuvo en cuenta el estar en terapia psicológica al momento de la aplicación de la prueba y estar diagnosticado por un profesional en salud mental (psicólogo clínico) con depresión o estado de ánimo bajo. En la descripción general, se identifica que el $72 \%$ de las personas se encuentra entre el estrato 1 y el 3; el $60.6 \%$ era soltero y el resto casado (a), divorciado (as), viudo (a) o vive en unión libre; el $73.9 \%$ estudian o trabajan y el resto eran independientes, desempleados y pensionados $(2.6 \%)$; el $55.7 \%$ de los participantes procedían del departamento de Cundinamarca y un $10 \%$ de Boyacá y los restantes de diferentes zonas del país; el $88.2 \%$ participante en la investigación correspondió a población comunitaria y el restante pertenece a la población clínica.

\section{Instrumentos}

\section{Environmental Reward Observation Scale}

La EROS (Armento \& Hopko, 2007) es un instrumento de 10 ítems que evalúa el grado de recompensa que aporta el entorno. Los ítems evalúan el incremento de la conducta y el afecto positivo que surge como consecuencia de vivir experiencias reforzantes del entorno (Anexo A). En la construcción de los ítems, se tuvieron en cuenta los tres criterios de Lewinsohn (1975) respecto a lo que representa el constructo "refuerzo positivo contingente a la respuesta": (1) el número de eventos potencialmente reforzantes, (2) la disponibilidad de reforzamiento en el entorno y (3) la conducta instrumental (habilidad) de un individuo para elicitar el reforzamiento. Los ítems de la EROS se responden usando una escala tipo Likert con cuatro alternativas de respuesta, que van desde 1 (totalmente en desacuerdo) a 4 (totalmente de acuerdo). El total se obtiene por la suma de los 10 ítems (directos e inversos) (Anexo B). La EROS posee una única dimensión, confirmada a través de tres estudios independientes (Armento \& Hopko, 2007).

\section{Escala Autoaplicada de Zung para Depresión}

Con el fin de identificar la validez divergente de la EROS, se utilizó la Escala Autoaplicada para la Depresión de Zung (1965), que evalúa el nivel de depresión en pacientes diagnosticados con algún tipo de trastorno afectivo. Cuenta con 20 reactivos que indican las cuatro características más comunes de la depresión: las actividades psicomotoras, las respuestas fisiológicas, el afecto dominante y otras perturbaciones. Consta de diez preguntas elaboradas de forma positiva y otras diez, de forma negativa. Cada pregunta se evalúa en una escala de 1-4 donde: 1 es poco tiempo; 2, algo de tiempo; 3, una buena parte del tiempo y 4 , la mayor parte del tiempo, y sus rangos de calificación van de 20 a 80 .

\section{Procedimiento}

El proceso de la investigación se realizó según las siguientes fases:

Fase I. Autorización:

El objetivo de la primera fase fue obtener la autorización para la utilización de las escalas con fines académicos e investigativos. Dicho permiso se encontró disponible en la World Wide Web para uso de la versión en inglés. Para la utilización de la adaptación española, se redactó un correo solicitando permiso a los investigadores, los cuales dieron su aval por este mismo medio. Para el pilotaje de este estudio se utilizó la versión en español traducida por Barraca-Mairal y Pérez-Álvarez (2010). Simultáneamente, se generó el consentimiento informado. En dicho formato, se aclararon aspectos como el objetivo de la escala aplicada, la confidencialidad y la participación voluntaria de cada uno de los sujetos.

Fase II. Evaluación: 
En esta fase, se procedió a la evaluación por parte de jueces expertos. Siete profesionales en psicología con formación de maestría y conocimientos en el área de análisis conductual aplicado, AC y psicometría. Cada uno evaluó dos grandes categorías. La primera fue pertinencia, referida a la adecuación del contenido del ítem para la medición del constructo que se pretende medir (Pardo, 2006). Se contó con tres opciones de evaluación: 1) Esencial (E): el ítem mide el constructo que se pretende, lo que significa que el contenido del ítem es esencial para la medición del constructo; 2) Útil pero no esencial (U): implica que el ítem mide algún aspecto del constructo, sin embargo, el contenido es útil pero no es esencial para la medición y 3) No necesario $(\mathrm{NN})$ : el ítem $\mathrm{NO}$ mide el constructo o es completamente irrelevante para su medición.

La segunda categoría evaluada por los expertos se denominó estructura, que se refiere a la adecuación de la disposición interna del ítem, al grado de coherencia y claridad del contenido (Pasquali, 1998). Las dos opciones de calificación de esta categoría fueron: 1) Adecuado (A): el ítem mantiene los parámetros establecidos para el enunciado y las opciones de respuesta según su tipo de escala (Likert) y su redacción es clara; 2) No adecuado (NA): el ítem no sigue los parámetros establecidos para el tipo de escala y presenta dificultades para su comprensión.

Para valorar la validez de contenido a partir del proceso de validación por jueces expertos, se utilizó el índice de Kappa de Fleiss (1971), mediante el cual se identifica el porcentaje de acuerdo entre tres o más evaluadores, además de tener en cuenta los efectos del azar y la razón de validez de contenido (CVR), y así identificar si el ítem es esencial o no para la prueba, dadas las condiciones definidas en el formato de validación.

Fase III. Pilotaje:

En esta fase, se llevó a cabo el pilotaje con 25 estudiantes universitarios quienes respondieron la versión adaptada al español de la EROS. Se realizó una bitácora de aplicación en la cual se registraron todos los pormenores de la misma, posterior a la revisión y modificación propuesta por los jueces. Simultáneamente, se llevó a cabo un análisis cualitativo de los reactivos en el área de exploración del lenguaje de la prueba (Cohen \& Swerdlik 2001), con ocho estudiantes de maestría en Psicología Clínica para verificar su grado de comprensión. En esta fase, se realizaron las modificaciones pertinentes según el pilotaje en el cuestionario de datos sociodemográficos y la revisión de la comprensión de ítems.

Fase IV. Permisos:

Durante esta fase, se realizaron los trámites relacionados con la consecución de permisos para aplicación en la muestra "clínica". Se solicitó la autorización de doce centros de psicología clínica de las diferentes universidades de Bogotá, además de contar con la colaboración de 25 psicólogos clínicos en práctica privada.

Fase V. Generación de prueba:

Para la aplicación de la escala a la muestra comunitaria y clínica, se adaptó la prueba en medio magnético mediante la herramienta Web Typeform; en la planilla general de la prueba, se adjuntaron el consentimiento informado, el cuestionario de datos sociodemográficos, la Enviroment Reward Observation Scale y la Escala Autoaplicada para la Depresión de Zung.

Frente a cada uno de los apartados, se adjuntaron instrucciones de diligenciamiento con el fin de garantizar la adecuada respuesta a las escalas. Para la muestra "clínica", se radicaron cartas de permisos en los centros de psicología de la ciudad de Bogotá; posteriormente, se envió vía correo electrónico el enlace de la plataforma a los directores de los centros y a los profesionales del área clínica, con el fin de que cada uno socializara con los pacientes la investigación y en últimas en el transcurso de las sesiones terapéuticas diligenciar las pruebas, a la cual se pudo acceder desde computadores, tabletas y smartphones.

Fase VI. Unificación de datos:

En esta fase, se unificó la base de datos que arroja la plataforma, se revisaron los datos y ajustaron para su análisis estadístico y psicométrico en el paquete SPSS versión 22.0. El ajuste mencionado se centró en la unificación de cada una de las puntuaciones, la eliminación de algunos datos que no cumplían 
con las características de la investigación, la calificación de las puntuaciones negativas (ítems de calificación inversa), tanto en la EROS como en el inventario de Zung y la sumatoria total, de dichas puntuaciones. Se procedió a generar el plan de análisis de la investigación, el cual estuvo compuesto por análisis por parte de jueces, análisis estadísticos básicos, análisis factorial exploratorio con y sin rotación VARIMAX, consistencia interna de la prueba, validez divergente y validez discriminante.

Para los análisis estadísticos básicos se obtuvieron las medidas de tendencia central, medidas de dispersión y medidas de forma y asimetría. En los análisis psicométricos se obtuvieron la frecuencia de respuesta y el análisis de discriminación para cada ítem. Seguidamente, se contrastó la información del análisis psicométrico con los resultados del análisis de jueces expertos, lo que permitió identificar las diferentes fuentes de validez de contenido. También se determinó la consistencia interna de la escala y la correlación entre los ítems a partir del cálculo del índice alfa de Cronbach. Posteriormente, se realizó el análisis factorial con y sin rotación VARIMAX para validez de constructo. Por último, y con el fin de identificar la validez divergente, se calculó la correlación de las puntuaciones totales de la EROS con la SDS en su versión original y para la validez discriminante, mediante la prueba t para muestras independientes.

\section{Resultados}

\section{Análisis por jueces}

Con respecto a la razón de validez de contenido, en los resultados del CVR se identificó que los ítems 3,5 y 8 presentaron índices negativos, lo que implica que tales ítems son necesarios pero no esenciales para la versión final de la prueba.

En el Kappa de Fleiss se obtuvo un índice de 0.68 , que indica un nivel de acuerdo sustancial en relación con lo postulado por Landis y Koch (1977).
Durante la aplicación del pilotaje, se llevó a cabo el registro de la sesión en una bitácora; con respecto a este soporte, cabe recalcar que en el transcurso de la aplicación no se presentaron inquietudes con respecto a prueba.

TABLA 1

Razón de validez de contenido

Razón de validez de contenido

\begin{tabular}{|c|c|}
\hline Ítems & $\begin{array}{l}\text { Razón de validez } \\
\text { de contenido }\end{array}$ \\
\hline IT 1 & 0.43 \\
\hline IT 2 & 0.43 \\
\hline IT 3 & -0.43 \\
\hline IT 4 & 0.43 \\
\hline IT 5 & -0.43 \\
\hline IT 6 & 0.43 \\
\hline IT 7 & 0.14 \\
\hline IT 8 & -0.14 \\
\hline IT 9 & 0.14 \\
\hline IT 10 & 0.43 \\
\hline
\end{tabular}

Fuente: elaboración propia

\section{Análisis descriptivos básicos}

La distribución de respuesta por cada ítem se presenta en la Tabla 2. Se puede observar cuáles fueron las opciones de respuesta con la mayor y menor marcación de la población, en términos de porcentaje. 
TABLA 2

Distribución de respuesta por ítem

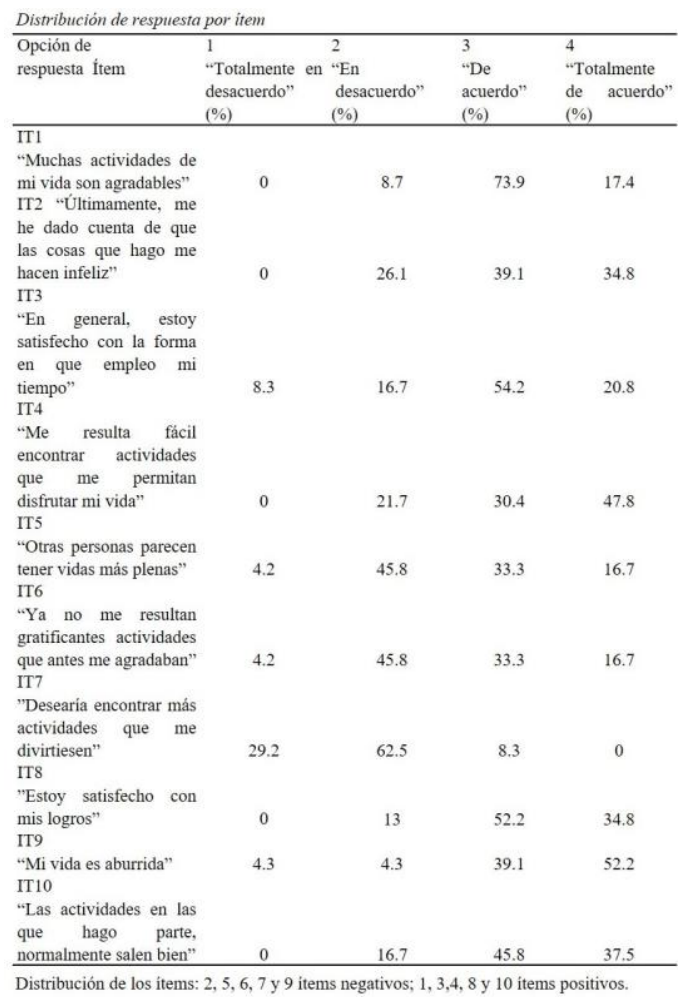

Fuente: elaboración propia

Por otra parte, para establecer la discriminación de ítems se utilizó el coeficiente de discriminación ítem-prueba corregido. Dado los resultados obtenidos se tiene que los ítems 3,5 y 6 presentan la correlación por debajo de 0.4 , mientras que los restantes están por encima; es relevante mencionar que el ítem 10 es el que presenta mayor correlación con la prueba (Tabla $3)$.
TABLA 3

Índice de discriminación

Índice de discriminación

\begin{tabular}{cc}
\hline İtems & \multicolumn{2}{l}{$\begin{array}{l}\text { Índice de } \\
\text { Discriminación } \\
\text { corregido }\end{array}$} \\
\hline IT 1 & 0.43 \\
IT 2 & 0.5 \\
IT 3 & 0.31 \\
IT 4 & 0.68 \\
IT 5 & 0.26 \\
IT 6 & 0.33 \\
IT 7 & 0.45 \\
IT 8 & 0.52 \\
IT 9 & 0.46 \\
IT 10 & 0.62 \\
\hline
\end{tabular}

Fuente: elaboración propia

Consistencia interna-confiabilidad de la
prueba

Se obtuvo un \# de Cronbach de 0.87 , valor que se encuentra dentro del rango esperado para la confiabilidad en este tipo de escalas.

\section{Validez de constructo}

\section{Análisis factorial exploratorio}

Teniendo en cuenta los resultados del coeficiente de correlación, se procedió a examinar la dimensionalidad del inventario, para lo cual se llevó a cabo un análisis factorial exploratorio. Antes de realizar dicho análisis, se calcularon la medida de adecuación muestral de KaiserMeyer-Olkin (KMO) y el test de esfericidad de Bartlett. El índice KMO mostró un valor de 0.92, el cual se considera aceptable, y respecto al test de Bartlett, con 45 grados de libertad se obtuvo un valor de significancia de 0 lo que permite establecer que los datos son susceptibles a la realización del análisis factorial.

En primer lugar, considerando el criterio de extracción de factores de Kaiser se encontraron 
Mario Andrés Valderrama-Díaz, Javier Mauricio Bianchi-Salguero, Javier Andrés Villalba-Garzón.

nueve factores que explican el $96.13 \%$ de la varianza, por ende existe un $3.86 \%$ de varianza no explicada en el modelo. Por otra parte, se identifican dos factores que explican el $56.93 \%$ de la varianza total y abarcan todos los ítems de la prueba.

\section{TABLA 4}

Porcentaje de varianza por factor

\begin{tabular}{ccc} 
Porcentaje de varianza por factor \\
\hline \multicolumn{3}{c}{$\begin{array}{c}\text { Auto-valores iniciales } \\
\text { \% de varianza }\end{array}$} \\
Factor & 46.81 & 46.81 \\
\hline 1 & 10.12 & 56.93 \\
2 & 6.81 & 63.73 \\
3 & 6.51 & 70.23 \\
4 & 5.87 & 76.1 \\
5 & 5.57 & 81.68 \\
6 & 5.31 & 86.98 \\
7 & 4.78 & 91.77 \\
8 & 4.35 & 96.13 \\
9 & 3.86 & 100 \\
10 & &
\end{tabular}

Fuente: elaboración propia

En la Tabla 5, se presentan a continuación los pesos factoriales para cada uno de los ítems respecto al Factor 1 denominado noción de refuerzo media ambiental.

\section{TABLA 5}

\section{Matriz factorial}

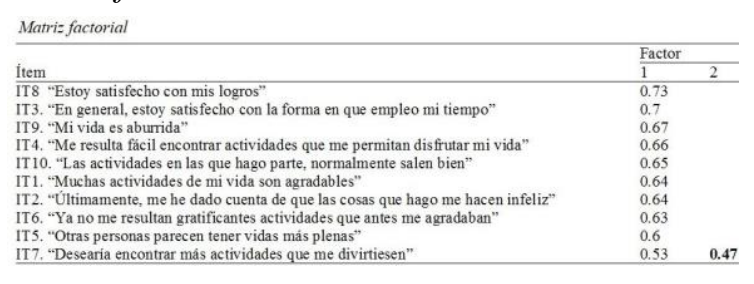

Fuente: elaboración propia

De acuerdo con la Tabla 6, se evidencia que el ítem 7 ("Desearía encontrar más actividades que me divirtiesen") se ubica en dos factores. La matriz presentada hace referencia a la arrojada sin rotación VARIMAX, ya que luego de realizar dicho procedimiento, se identificó que los ítems se desajustan, en otras palabras, se encuentra una mayor agrupación de los factores y una mejor carga factorial sin la rotación VARIMAX.

\section{Validez divergente}

Según los resultados del coeficiente de correlación lineal de Pearson se obtuvo una correlación negativa y moderada ( $r=-0.67)$, una significancia de 0 y 103 grados de libertad, lo cual indica que a mayor puntuación en la prueba de noción de refuerzo medio ambiental (medida de la EROS), se evidencian puntuaciones bajas en el inventario de ZUNG (bajo nivel de depresión).

\section{Validez discriminante}

Otro de los análisis realizados fue la comparación del grupo clínico y comunitario. Para esta comparación, se realizaron pruebas paramétricas siempre y cuando se cumplieran los supuestos para el uso de las mismas. Respecto al supuesto de normalidad, se utilizó Prueba de Kolmogorov-Smirnov, la cual indica que los datos y las variable de interés sigue una distribución normal $(Z=0.06)$. Para validar el supuesto de homogeneidad de las varianzas, se utilizó la Prueba de Levene, mediante la cual se observa que las varianzas son homogéneas (F $=8.85$ ), además una significancia de 0. Dado los anteriores resultados, se procedió a utilizar la prueba t para dos muestras independientes, la cual permite identificar diferencias significativas entre las medias de dos grupos. Según los resultados de la prueba estadística con 573 grados de libertad, se obtuvo una $\mathrm{t}=11.19 \mathrm{y}$ significancia de 0 , lo cual indica que existe evidencia suficiente para afirmar que hay diferencias significativas entre ambos grupos.

Por otra parte, el tamaño del efecto tiene un valor de 1.18 según la medida del $\mathrm{G}$ de Hedges, lo que indica que el tamaño del efecto es grande y en una distribución normal existe una probabilidad de 0.88 de que la diferencia entre los dos grupos se deba a la categoría diagnóstica utilizada, en este caso la depresión o el bajo estado de ánimo. 


\section{Discusión y conclusiones}

El objetivo de esta investigación fue presentar las propiedades psicométricas de la EROS, instrumento creado por Armento y Hopko (2007) y validado en población española por BarracaMairal y Pérez-Álvarez (2010), que es un instrumento válido y fiable para obtener medidas sobre la noción de refuerzo medioambiental de los consultantes, y que puede interpretarse como una escala que relaciona bajas puntuaciones con la presencia de un bajo nivel o noción de refuerzo medioambiental y por ende un elevado nivel de depresión o estado de ánimo bajo. Esto tomando como base la correlación presente entre la EROS y el SDS-ZUNG.

La medición de este constructo guarda una estrecha relación y resulta importante en los modelos explicativos de la depresión y los procesos adyacentes de la AC, técnica que consiste en el incremento de dicha noción mediante la implementación de tareas o actividades que aumenten la interacción del sujeto con su contexto. Si bien la intervención facilita el acceso a las recompensas del medio ambiente, el proceso carece de utilidad si el individuo no percibe dicha mejora, razón por la cual es importante sumar otras formas de medida diferentes al autoinforme.

Al realizar los estudios estadísticos y psicométricos de la EROS, se hace evidente una alta confiabilidad, así como evidencias de validez importantes con una muestra total de 575 participantes, dispersos en un amplio rango de edad, con presencia en 22 departamentos del país y presencia de población clínica y no clínica, con un \# de 0.87 .

Respecto a la confiabilidad de la prueba, según los resultados del índice \# de Cronbach (\# = 0.87), se puede afirmar que el instrumento cuenta con un elevado nivel de consistencia interna. El índice de confiabilidad es mayor al presentado en los estudios españoles de Barraca-Mairal y Pérez-Álvarez y el estudio estadounidense original de Armento y Hopko (0.86 y 0.85, respectivamente).

De acuerdo al conjunto de análisis psicométricos y estadísticos presentados en el apartado de resultados, es claro que la Environment Reward Observation Scale es un instrumento que cuenta con evidencias de validez, lo cual lo hace una estrategia apropiada para evaluar la noción de refuerzo medioambiental en población clínica y comunitaria, brindado una explicación más cercana al comportamiento y no enfocándose en una descripción o categorización de la problemática del consultante.

En cuanto a las evidencias de validez (constructo, discriminante y divergente), se encuentra que con respecto a la validez de constructo, los resultados de la prueba en términos factoriales son similares a los dos estudios base, se reporta una consistencia unidimensional de la escala, esta dimensión se adapta a los postulados teóricos sobre la depresión y la pérdida de refuerzo positivo contingente a la respuesta (Ferster, 1973; Lewinsohn, 1975; Seligman, 1975; Kanter, Cautilli, Busch, \& Baruch, 2011).

Un aspecto relevante en cuanto al análisis factorial, es la carga del ítem 7 ("Desearía encontrar más actividades que me divirtiesen") el cual se ubica en dos factores; si bien existe una carga factorial, no se cuenta con evidencia empírica suficiente de la presencia de un segundo factor, ya que según Hair, Black, Babin y Anderson (2010) para considerar la existencia de un factor se debe tener como mínimo tres ítems que posean peso en el componente, además de tener en cuenta que el factor uno posee una mayor carga factorial en comparación al segundo. En otras palabras, este ítem estaría midiendo algo más; teniendo en cuenta la redacción del ítem, este podría responder o estar también enfocado en la evaluación del área vital recreación e intereses, planteada por LeJuez, Hopko, Acierno, Daughters y Pagoto (2011) que tiene relación entre estado de ánimo y conductas reforzantes. Esta relación ha sido planteada por teóricos como Hopko, Armento, Cantu, Chambers \& LeJuez. (2003) que mencionan la diferencia entre la realización de una actividad y el disfrute de la misma, ya que muchas personas desarrollan actividades placenteras que terminan presentado funciones de evitación y escape al 
Mario Andrés Valderrama-Díaz, Javier Mauricio Bianchi-Salguero, Javier Andrés Villalba-Garzón.

estado de ánimo bajo, que no necesariamente representa una baja o alta noción de refuerzo medioambiental.

Por otra parte, se evidencia una correlación aceptable, inversamente proporcional (validez divergente) entre la EROS y la escala para la depresión de Zung, la cual es un indicador significativo de la validez de criterio. En esta línea, se identifica que la aplicación de la escala EROS con una prueba relacionada con la medición de la depresión (en este caso la escala para la depresión de Zung) permite la identificación de mejoras o modificaciones en los síntomas depresivos, en otras palabras, bajas puntuaciones en el inventario de Zung se relacionan con puntuaciones altas en la EROS, lo cual podría interpretarse como una mejora en la percepción o noción de refuerzo medioambiental. Se pasa de esta manera a integrar un grupo de pruebas psicológicas que fortalecen la evaluación multimétodo $\mathrm{y}$ multifuente de los postulados teóricos de la depresión desde una visión contextual y por ende de la AC una alternativa de intervención.

No puede dejar de mencionarse que la EROS original y su versión española han sido utilizadas en un variado número de investigaciones con tratamientos de Activación Conductual (Barraca-Mairal, 2010; Carvalho et al., 2011; Daughters et al., 2008; Gawrysiak et al., 2009), y se han identificado en estos documentos, así como en la presente investigación, como una medida confiable y válida para la evaluación de la noción de refuerzo medioambiental así como de los postulados de la AC.

\section{Referencias}

Armento, M. E. A., \& Hopko, D. R. (2007). The Environmental Reward Observation Scale (EROS): Development, validity, and reliability. Behavior Therapy, 38(2), 107-119.

Barraca-Mairal, J. (2010). Aplicación de la Activación Conductual en un paciente con sintomatología depresiva. Clínica y Salud, 21(2), 183-197.
Barraca-Mairal, J., \& Pérez-Álvarez, M. (2010). Adaptación española del Environmental Reward Observation Scale (EROS). Ansiedad y Estrés, 16(1), 95-107. Recuperado de http://jorgebarraca.com/wp-content/upl oads/Adaptaci\%C3\%B3nespa\%C3\%B1ol adelEnvironmentalRewardObservationSca le-EROS.pdf

Beltrán, M. del C., Freyre, M. Á., \& HernándezGuzmán, L. (2012). El Inventario de Depresión de Beck: su validez en población adolescente. Terapia Psicológica, 30, 5-13. http://dx.doi.org/10.4067/S0718-480 82012000100001

Bianchi-Salguero, J. M., \& Muñoz-Martínez, A. M. (2014). Activación Conductual: revisión histórica, conceptual y empírica. Psychologia: Avances de la Disciplina, 8(2), 83-93.

Busch, A. M., Kanter, J. W., Callaghan, G. M., Baruch, D. E., Weeks, C. E., \& Berlin, K. S. (2009). A microprocess analysis of Functional Analytic Psychotherapy's mechanism of change. Behavior Therapy, 40, 280-290. http:// dx.doi.org/10.1016/j.beth.2008.07.003

Carvalho, J. P., Gawrysiak, M. J., Hellmuth, J. C., McNulty, J. K., Magidson, J. F., LeJuez, C. W., \& Hopko, D. R. (2011). The reward probability index: Design and validation of a scale measuring access to environmental reward. Behavior Therapy, 42, 249-262. ht tp://dx.doi.org/10.1016/j.beth.2010.05.004

Carvalho, J. P., \& Hopko, D. R. (2009). Treatment of a depressed breast cancer patient with problem-solving therapy. Clinical Case Studies, 8, 263-276. http://dx .doi.org/10.1177/1534650109337732

Cohen, R. J., \& Swerdlik, M. E. (2001). Pruebas y evaluación psicológicas: introducción a las pruebas y a la medición (4. ${ }^{\mathrm{a}}$ ed.). México: McGraw-Hill.

Daughters, S. B., Magidson, J. F., Schuster, R. M., \& Safren, S. A. (2010). ACT HEALTHY: A combined cognitivebehavioral depression and medication adherence treatment for HIV-infected 
substance users. Cognitive and Behavioral Practice, 17, 309-321. http://dx.doi.org/10. 1016/j.cbpra.2009.12.003

Daughters, S. B., Sargeant, M. N., Bornovalova, M. A., Gratz, K. L., \& LeJuez, C. W. (2008). The relationship between distress tolerance and antisocial personality disorder among male inner-city treatment seeking substance users. Journal of Personality Disorders, 22, 509-524. http:// dx.doi.org/10.1521/pedi.2008.22.5.509

Dimidjian, S., Martell, C., Addis, M., \& HermanDunn, R. (2008). Behavioral activation for depression. En D. H. Barlow (Ed.), Clinical handbook of psychological disorders (4.a ed., pp. 328-364). Nueva York: Guilford Press.

Ferster, C. B. (1973). A functional analysis of depression. American Psychologist, 28, 857-870.

Fleiss, J. L. (1971). Measuring nominal scale agreement among many raters. Psychological Bulletin, 76, 378-382. http:/ /dx.doi.org/10.1037/h0031619

Gamero, R. R., Vucínovich, N., \& Oñate, S. P. (2011). Group psychotherapy for men with chronic major depressive disorder in a community mental health unit. Psychology in Spain, 15(1), 1-8. Recuperado de http://www.psychologyins pain.com/content/full/2011/15001.pdf

Gawrysiak, M., Nicholas, C., \& Hopko, D. (2009). Behavioral activation for moderately depressed university students: Randomized controlled trial. Journal of Counseling Psychology, 56, 468-475. http: //dx.doi.org/10.1037/a0016383

Hair, J., Black, W., Babin, B., \& Anderson, R. (2010). Multivariate data analysis. Nueva York: Prentice Hall.

Hopko, D. R., Armento, M. E. A., Cantu, M. S., Chambers, L. L., \& LeJuez, C. W. (2003). The use of daily diaries to assess the relations among mood state, overt behavior, and reward value of activities. Behaviour Research and Therapy, 41(10), 1137-1148. http://dx.doi.org/10.1016/S000 5-7967(03)00017-2
Hugo-Mendoza, E. C. (2014). Aplicación de la terapia dialéctica conductual y terapia cognitivo-conductual: un caso comórbido de trastorno de personalidad límite (Tesis de pregrado no publicada). Universidad San Francisco de Quito, Colegio de Ciencias Sociales y Humanidades, Quito, Ecuador.

Kanter, J. W., Cautilli, J. D., Busch, A. M., \& Baruch, D. E. (2011). Toward a comprehensive functional analysis of depressive behavior: Five environmental factors and a possible sixth and seventh. International Journal of Behavioral Consultation and Therapy, 7, 5-14. http://dx.doi.org/10.1037/h0100055

Kanter, J. W., Mulick, P. S., Busch, A. M., Berlin, K. S., \& Martell, C. R. (2006). The Behavioral Activation for Depression Scale (BADS): Psychometric properties and factor structure. Journal of Psychopathology and Behavioral Assessment, 29, 191-202. http://dx.doi.org /10.1007/s10862-006-9038-5

Kanter, J. W., Rusch, L. C., Busch, A. M., \& Sedivy, S. K. (2008). Validation of the Behavioral Activation for Depression Scale (BADS) in a community sample with elevated depressive symptoms. Journal of Psychopathology and Behavioral Assessment, 31, 36-42. http://dx.doi.org/10 .1007/s10862-008-9088-y

Landis, J. R., \& Koch, G. G. (1977). The measurement of observer agreement for categorical data. Biometrics, 33, 159-174. http://dx.doi.org/10.2307/2529310

LeJuez, C., Hopko, D., Acierno, R., Daughters, S., \& Pagoto, S. (2011). Ten year revision of the brief behavioral activation treatment for depression: Revised treatment manual. Behavior Modification, 35, 111-161. http:/ /dx.doi.org/10.1177/0145445510390929

Lewinsohn, P. M. (1975). The behavioral study and treatment of depression. Progress in Behavior Modification, 1, 19-64.

MacPherson, L., Tull, M. T., Matusiewicz, A. K., Rodman, S., Strong, D. R., Kahler, C. W., \& LeJuez, C. W. (2010). 
Randomized controlled trial of behavioral activation smoking cessation treatment for smokers with elevated depressive symptoms. Journal of Consulting and Clinical Psychology, 78, 55-61. http://dx.d oi.org/10.1037/a0017939

Montero, I., \& León, O. (2007). A guide for naming research studies in psychology. International Journal of Clinical and Health Psychology, 7(3), 847-862.

Pardo, C. A. (febrero, 2006). Hablemos de validez. Taller realizado en el Primer Seminario Internacional de Evaluación de la Educación, Cartagena, Colombia.

Pasquali, L. (1998). Principios de elaboração de escalas psicológicas. Archives of Clinical Psychiatry, 25(5), 206-213.

Pérez-Álvarez, M. (2007). La activación conductual y la desmedicalización de la depresión. Papeles del Psicólogo, 28(2).

Ryba, M. M., LeJuez, C. W., \& Hopko, D. R. (2014). Behavioral activation for depressed breast cancer patients: The impact of therapeutic compliance and quantity of activities completed on symptom reduction. Journal of Consulting and Clinical Psychology, 82(2), 325-335. h ttp://dx.doi.org/10.1037/a0035363

Seligman, M. E. P. (1975). Helplessness: On depression, development, and death. Nueva York: Henry Holt \& Co.

Skinner, B. (1953). Science and human behavior. Nueva York: Macmillan.

Zung, W. W. K. (1965). A Self-Rating Depression Scale. Archives of General Psychiatry, 12, 63-70. http://dx.doi.org/10. 1001/archpsyc. 1965.01720310065008

\section{ANEXO A}

\section{Environmental Reward Observation Scale (EROS) Versión colombiana}
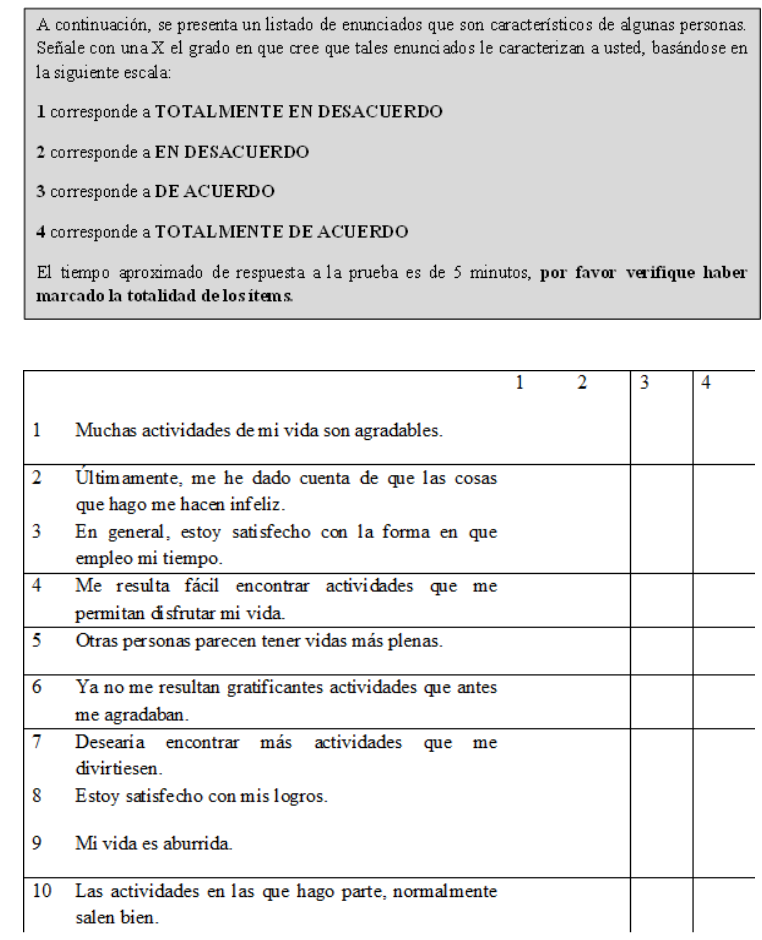

\section{ANEXO B}

\section{Versión colombiana (criterios de calificación)}

A continuación, se presenta un listado de enunciados que son característicos de algunas personas.
Señale con una $\mathrm{X}$ el grado en que cree que tales enunciados le caracterizan a usted, basándose en
la siguiente escala:
1 corresponde a TOTALMENTE EN DESACUERDO
2 corresponde a EN DESACUERDO
3 corresponde a DE ACUERDO
4 corresponde a TOTALMENTE DE ACUERDO
El tiempo aproximado de respuesta a la prueba es de 5 minutos, por favor verifique haber
marcado la totalidad de lositems.




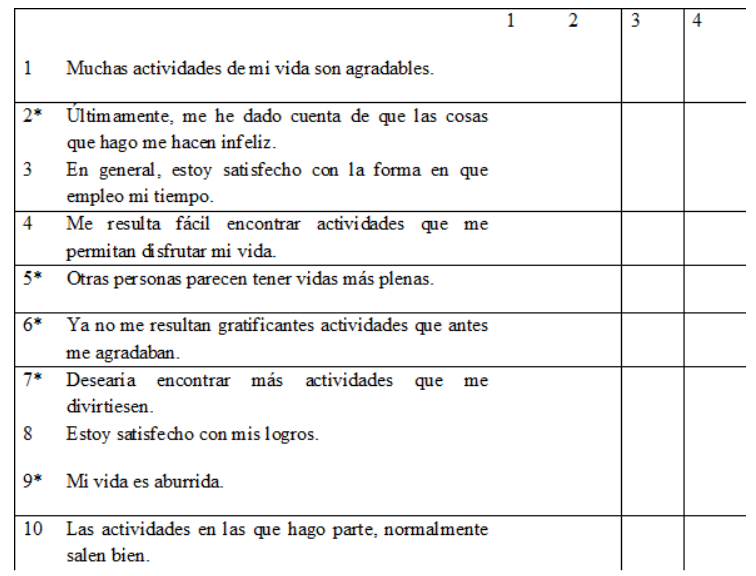

A mayor puntuación mayor noción de refuerzo del medio ambiente $=$ menor estado de ánimo bajo

Puntuación máxima 40, mínima 10.

De cara a la aplicación y calificación del instrumento, hay que tener en cuenta que los ítems $2,5,6,7$ y 9 se corrigen de forma inversa $(1=4 ; 2=3 ; 3=2 ; 4=1)$.

\section{Notas}

* Artículo de investigación. 